\title{
Una experiencia de educación emocional en el campamento urbano de verano de La Granja Escuela: una innovación en el ámbito del ocio educativo
}

\section{An emotional educational's experience at the La Granja's summer camp: an innovation in the field of leisure education}

\author{
Cristina Gutierrez Lestón ${ }^{1}$, Marta Eroles ${ }^{1}$, Nuria Perez Escoda ${ }^{2}$, Mercedes Reguant $^{2}$ \\ cristina@la-granja.net,marta@la-granja.net,nperezescoda@ub.edu,mercedesreguant@gmail.com \\ Investigación educativa ${ }^{1}$ \\ La Granja, Ability Training Center \\ Santa Maria de Palautordera \\ Barcelona, España \\ GROP, Grupo de investigación de Orientación ${ }^{2}$ \\ Psicopedagógica \\ Universitat de Barcelona \\ Barcelona, España
}

\begin{abstract}
Resumen- Son muy pocas las investigaciones sobre la capacidad educativa de las actividades de tiempo libre educativo infantil y juvenil (formación no reglada) que se celebran en periodo de vacaciones de verano. Así mismo tampoco son frecuentes los trabajos científicos sobre los programas de educación emocional en el ámbito del ocio educativo. En este marco, se presenta un estudio que pretende demostrar que el Método de educación emocional de La Granja en el ámbito de la formación no reglada y del ocio educativo, permite mejorar la competencia emocional de los niños y niñas participantes y disminuir los sus niveles de ansiedad. Se trata de la evaluación de un proyecto innovador desarrollado durante el verano de 2017 de manera práctica y vivencial, mediante juegos, dinámicas y actividades con un reto a conseguir, y donde se utilizan recursos como los caballos de doma natural, actividades de aventura en el bosque o animales de granja, para el desarrollo de competencias emocionales. La muestra la forman un total de 91 niños y niñas de edades comprendidas entre 8 y 12 años. Los resultados ponen en evidencia que en un periodo de cinco semanas es posible obtener mejoras considerables.
\end{abstract}

Palabras clave: educación emocional, campamento urbano de verano, educación no formal, competencias emocionales, innovación educativa.

Abstract- There are not many researches on the educational capacity of educational leisure activities (non-formal education) for kids and teenagers, which are held during the summer vacation period. Also scientific works on emotional education programs are not frequent in the field of educational leisure. In this framework, this study is presented that aims to demonstrate that the La Granja's Emotional Education Methodology, in the field of non-formal education and educational leisure, allows the kids to improve the emotional competences children and decrease anxiety and stress levels in them. It's the evaluation of an innovative project developed during the summer of 2017, during 5 weeks, in a practical and livid way, through games, dynamics and activities with a challenge to be achieved, and where resources such as natural dressage horses are used, Adventure activities in the forest or with the animals in the farm are lived, etc., for the development of emotional competencies. The sample is made up of 91 boys and girls aged from 8 to 12 years old. The results show that in a period of five weeks it is possible to obtain considerable improvements.

Keywords: emotional education, summer camp, non-formal education; emotional competencies; educational innovation.

\section{INTRODUCCIÓN}

De acuerdo con Gil Cantero (1993) las actividades de ocio en el ámbito de la educación no formal impactan educativamente en los niños y en los jóvenes. No obstante es importante que los participantes tomen conciencia del valor y la importancia de utilizar el tiempo libre de manera positiva y activa para su proceso de crecimiento y maduración. Así entendemos la educación no formal como un proceso intencional de propuestas educativas donde las actividades, o bien no responden a los métodos habituales de la educación formal, o bien -ya sean actividades realizadas dentro o fuera del recinto escolar- no pertenecen a la enseñanza reglada, los requisitos académicos de acceso y continuación en la misma (Trilla, 1986).

Como actividades educativas en el tiempo libre han surgido en los últimos años muchas y diversas iniciativas entre las que destacaremos los campamentos o centros educativos donde de acuerdo con Mellado y Mellado (2006) se genera un clima propicio de vivencias, ambiente lúdico y al aire libre donde se pueden conseguir muchos objetivos pedagógicos que cuestan más trabajar en el marco de la enseñanza formal.

Hay de muchos tipos de campamentos urbanos, desde los más conocidos que probablemente sean los campus deportivos, a otras opciones pensadas como parque temático, centros de idiomas, centros de multi-aventura, etc. y propuestas innovadoras como el campamento urbano emocional que nos ocupa. 
Un campamento urbano es una experiencia educativa de primer orden caracterizado por tener una duración relativamente corta, en un ambiente o medio desconocido donde no hay profesores entendidos de forma tradicional y donde la familia juega un papel bastante secundario. No obstante, sus efectos suelen dejar una huella vital importante y contribuyen indiscutiblemente en su proceso madurativo.

Evaluar una experiencia de educación emocional dentro del ámbito de las actividades de ocio (educación no formal) infantil y juvenil durante los campamentos urbanos de verano en España es un hecho inédito y casi inaudito en la investigación científica.

En este trabajo se presentan los resultados de la evaluación del método de Educación Emocional de La Granja aplicado a un proyecto de campamento urbano de verano. La Granja Escuela de Santa María de Palautordera se define como un espacio que utiliza la educación emocional como herramienta para educar, y donde la convivencia se convierte en una experiencia positiva para el recuerdo de los que pasan por ella.

\section{CONTEXTO}

En este trabajo se estudian los efectos del campamento urbano emocional de verano entendido como proyecto innovador desarrollado durante el verano de 2017 de manera práctica y vivencial, mediante juegos, dinámicas y actividades con un reto a conseguir, y donde se utilizan recursos como los caballos de doma natural, instalaciones de aventura en el bosque o animales de granja.

El casal de verano se desarrolló entre el 25 de junio y el 28 de Julio a horario de 9:30 a 17:30 de lunes a viernes. Cada semana se trabajaba de forma intencional sobre una competencia emocional. Durante las mañanas los niños y niñas participantes realizaban actividades educativas explícitamente pensadas para desarrollar la competencia emocional que correspondía, y por las tardes, se realizaban actividades con un componente más lúdico y sin el componente explícito formativo como ir a la piscina, contar cuentos, participar en los juego de agua, hacer actividades con las que también se velaba por el desarrollo de hábitos de vida saludable, como ordenar el espacio de las habitaciones, revisar las mochilas siendo responsable de su ropa, además de tener un espacio de tranquilidad después de comer para hacer los deberes (cuaderno de verano).

\section{DESCRIPCIÓN}

El programa educativo emocional prevé el desarrollo en las 5 competencias definidas por Bisquerra i Pérez (2007, 9-12):
1. Consciencia emocional
2. Regulación emocional
3. Autonomía emocional
4. Competencias sociales
5. Competencias para la vida y el bienestar

emocionales y competencias sociales se fueron trabajando según detalla la tabla 1 :

Tabla 1. Contenidos de educación emocional trabajados en función de los grupos y las semanas.

\begin{tabular}{c|cc}
\hline Semana/ciclo & Ciclo medio $3^{\circ}-4^{\circ}$ & Ciclo Superior:5 $5^{\circ}-6^{\circ}$ \\
\cline { 2 - 3 } Semana 1 & Autonomía & Autoconocimiento \\
Semana 2 & Autoestima & Confianza \\
Semana 3 & Confianza & Autonomía \\
Semana 4 & Competencias & Responsabilidad y \\
Semana 5 & Sociales & creatividad \\
\hline
\end{tabular}

\section{A. Método La Granja}

El método utilizado para educar a las competencias emocionales es totalmente práctico y vivencial con un alto impacto emocional en los niños y jóvenes, el cual se ha basado en el ensayo, prueba, acierto y error con miles de niños de todas las edades ( 3 a 17 años) y después de 12 años de experiencia.

El Método (Figura 1) se enmarca en el marco teórico de Bisquerra y Pérez (2007) y se basa en realizar actividades Outdoor Training proponiendo un reto con la intención de emocionar, ya que hemos observado a lo largo de los años que sin emoción, no hay interiorización de los aprendizajes en los niños (Krathwohl, 2002). Además, se lanzan estímulos positivos de manera continua para provocar la emoción más motivadora que tenemos los humanos: la alegría, la cual ayuda a activar los niños y jóvenes, incluso aquellos más apáticos. Se persigue potenciar el resultado gracias al alto rendimiento durante el programa que se les propone, con actividades cortas que eviten el aburrimiento y con una actitud por parte del educador de concentración y conciencia plena para aprovechar todo lo que pasa y todo lo que falta durante cada actividad. Como complemento, las herramientas del coaching, como el arte de hacer preguntas y el feed back permiten al participante tomar consciencia, interiorizar y anclar los conocimientos derivados del paso por el centro emocional y la aplicación del Método.

La metáfora también forma parte del Método pues ayuda a trabajar desde el inconsciente de cada niño facilitando el entendimiento, y con la transferencia de aquello que pasa en la actividad a aspectos de su vida en casa o en la escuela, se consigue ampliar y trascender el aprendizaje encontrándole un sentido resonante para cada uno de los participantes.

A todo esto, hay que añadir que la naturaleza, el bosque y los caballos de doma natural son otros elementos del Método La Granja que contribuyen a serenar los niños y jóvenes, ya que, desde hace unos años, nunca habíamos observado tanta aceleración en ellos. Es necesario serenarlos para que puedan acercarse al autoconocimiento y la conciencia emocional, y así obtener resultados de mejora en las competencias emocionales.

Los grupos de niños y niñas se organizaban por edades y tomando como referente los ciclos de educación primaria: ciclo medio $\left(3^{\circ}\right.$ y $\left.4^{\circ}\right)$ y ciclo superior $\left(5^{\circ}\right.$ y $\left.6^{\circ}\right)$. Las competencias 


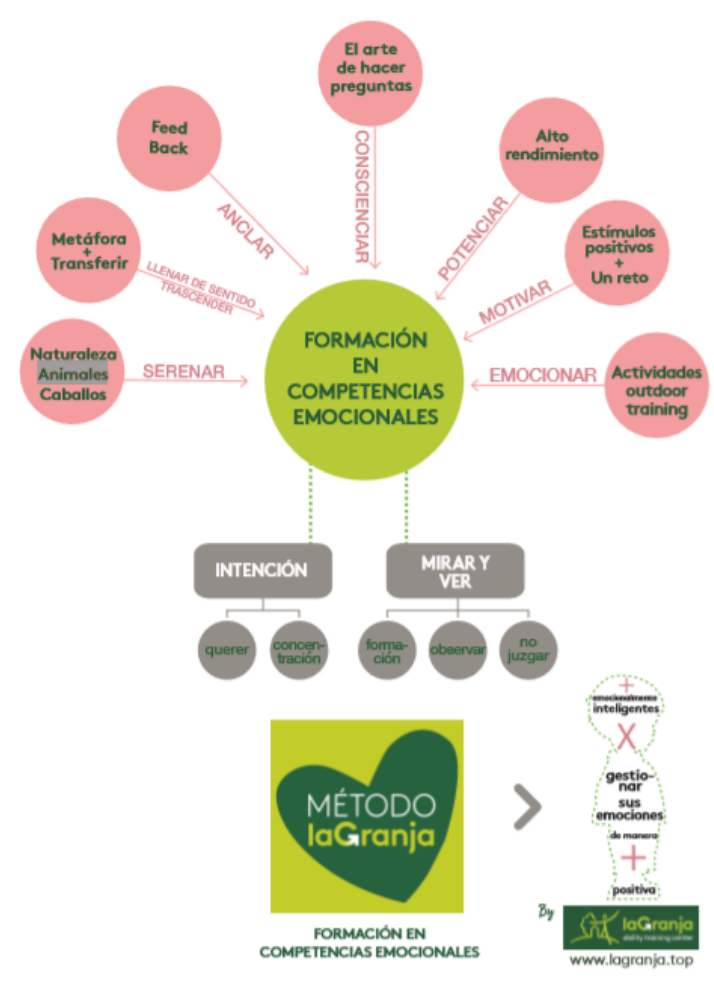

Figura 1. Representación del Método la Granja

\section{B) Estudio realizado}

Con el objetivo de evaluar los efectos del paso por el campamento emocional de verano se ha realizado un estudio cuasi-experimental en el que se estudiaba la variación en los niveles de competencia emocional y de ansiedad de los niños y niñas entre 8 y 12 años entre la situación inicial y final a la participación en el centro.

Los participantes no estuvieron escogidos al azar, sino que son los niños y niñas inscritos en los centros de verano por sus padres. En todo momento se ha respetado la confidencialidad de los datos y se han respetado los estándares éticos requeridos en el trabajo con seres humanos. Todas las familias han sido informadas y han tenido derecho a ejercer la oposición a la participación de sus hijos o hijas en la investigación.

La muestra estuvo formada por 91 niños y niñas con edades comprendidas entre los 8 y 12 años estudiantes de ciclo medio y superior de educación primaria. Puede verse la distribución de la muestra en la siguiente tabla:

Tabla 2. Distribución de la muestra por edad y género

\begin{tabular}{|c|c|c|c|c|c|}
\hline & & \multicolumn{4}{|c|}{ Sexo } \\
\hline & & $\begin{array}{l}\text { Ma } \\
\text { sc. }\end{array}$ & $\begin{array}{l}\mathrm{Fe} \\
\mathrm{m} .\end{array}$ & Total & $\%$ Total \\
\hline \multirow[t]{6}{*}{ Edad } & 8 & 5 & 3 & 8 & $8,8 \%$ \\
\hline & 9 & 14 & 12 & 26 & $28,6 \%$ \\
\hline & 10 & 6 & 10 & 16 & $17,6 \%$ \\
\hline & 11 & 19 & 10 & 29 & $31,9 \%$ \\
\hline & 12 & 7 & 5 & 12 & $13,2 \%$ \\
\hline & Total & 51 & 40 & 91 & \\
\hline
\end{tabular}

Para recoger datos sobre los niveles de competencia emocional se ha aplicado el cuestionario de desarrollo emocional para niños (CDE-9_13) elaborado por el GROP (Grupo de investigación en Orientación Psicopedagógica) de la Universidad de Barcelona de acuerdo con el marco teórico de Bisquerra y Pérez (2007). Se trata de una escala de tipo Likert de once puntos que consta de 38 ítems y su índice de consistencia interna según el coeficiente alfa de Cronbach es de 0.85 . Ofrece una puntuación global de la competencia emocional y puntuaciones parciales para cada una de las dimensiones: conciencia, regulación, autonomía, competencia social y competencias para la vida y el bienestar.

Para evaluar los cambios en la disminución de la ansiedad se ha utilizado el Cuestionario de Autoevaluación Ansiedad Estado / Rasgo en niños elaborado por Spielberger, Gorsuch y Lushene, (1982) y comercializado por TEA ediciones. Este cuestionario está formado por dos escalas de 20 ítems cada una que evalúan respectivamente la ansiedad rasgo y la ansiedad estado.

Además de ello al finalizar la estancia en el centro se preguntó a los niños y niñas sobre los aprendizajes hechos en el campamento urbano y que consideraban útiles y por qué lo consideraban. Las respuestas a estas cuestiones han permitido completar y confirmar los resultados obtenidos con los cuestionarios, dándole la riqueza de la exploración de significados personales respecto a la experiencia.

\section{Resultados}

Todos los datos cuantitativos se han tratado utilizando el procesador estadístico SPSS v.21

\section{A. Impacto sobre las competencias emocionales}

Tanto en la competencia emocional total, como las cinco dimensiones de la competencia emocional: conciencia, regulación, autonomía, competencia social y competencias de vida y bienestar, las puntuaciones en la fase post-test, es decir después de su participación en el campamento urbano, han aumentado. Así pues, podemos suponer que el paso por el campamento ha tenido un efecto en la mejora de la competencia emocional y en cada una de las dimensiones que la integran.

No obstante, se ha procedido a aplicar pruebas estadísticas de contraste de medias para poder estar seguros de que las diferencias observadas son significativas.

Tras comprobar que la muestra cumple con los requisitos para la aplicación de pruebas paramétricas, es decir, se ajusta a la curva normal y sus varianzas son similares, se ha procedido a efectuar el contraste entre las medias de acuerdo con la prueba $\mathrm{T}$ de muestras relacionadas, tal como se presenta en la Tabla 3. Posteriormente se ha calculado la magnitud de los cambios.

Se observa que la mejora en la competencia emocional total se produce con un nivel de significación de $\mathrm{p}<, 000$ por lo que podemos afirmar que existen diferencias significativas entre el antes y el después del campamento en los niveles de competencias emocionales de los niños y niñas participantes. Estas diferencias son a favor de la situación final, es decir que ha habido una mejora de las competencias tal como se puede 
ver en la tabla, se ha pasado de un nivel 6,85 a un nivel 7,18 sobre 10 .

Tabla 3. Cambios en las competencias emocionales entre la situación inicial y final

\begin{tabular}{|c|c|c|c|c|}
\hline & Media & $\begin{array}{l}\text { Desviación } \\
\text { típ. }\end{array}$ & Sig. & d Cohen \\
\hline $\begin{array}{l}\text { Pre-Total Comp. } \\
\text { Emocional }\end{array}$ & 6,85 & 1,01 & \multirow[t]{2}{*}{0,000} & \multirow[t]{2}{*}{,31 } \\
\hline Postest_Total & 7,18 & 1,12 & & \\
\hline Pre-Consciencia & 7,36 & 1,44 & \multirow{2}{*}{0,051} & \multirow{2}{*}{,14 } \\
\hline Postest_Consciencia & 7,57 & 1,47 & & \\
\hline Pre-Regulación & 5,88 & 1,48 & \multirow{2}{*}{0,000} & \multirow{2}{*}{,34 } \\
\hline Postest_Regulación & 6,38 & 1,46 & & \\
\hline Pre-Autonomía & 6,88 & 1,32 & \multirow{2}{*}{0,002} & \multirow{2}{*}{ 27, } \\
\hline Postest_Autonomía & 7,27 & 1,28 & & \\
\hline Pre-Compsoci & 6,62 & 1,60 & \multirow{2}{*}{0,001} & \multirow{2}{*}{,29 } \\
\hline Postest_Compsoci & 7,09 & 1,63 & & \\
\hline Pre-Cvida & 7,57 & 1,25 & \multirow{2}{*}{0,400} & \multirow{2}{*}{,07 } \\
\hline Postest_Cvida & 7,67 & 1,47 & & \\
\hline
\end{tabular}

Cuando analizamos el progreso en las diferentes dimensiones evaluadas, se observa que en todos los casos menos en competencias de vida y bienestar y conciencia emocional los cambios apreciados son estadísticamente significativos con una probabilidad que oscila entre $\mathrm{p}<, 05 \mathrm{y} \mathrm{p}<, 001$, también a favor de la situación final, es decir indicando una mejora en el nivel de competencia. En el caso de las competencias de vida y bienestar y conciencia emocional, aunque las diferencias no son estadísticamente significativas $\mathrm{p}=, 400 \mathrm{y}, 051$ respectivamente, si se observa que la tendencia de las puntuaciones indican un ligero progreso en positivo.

Una vez detectadas las diferencias significativas se ha procedido a calcular la magnitud de estos cambios, para ello se ha estudiado la $d$ de Cohen. De acuerdo con los resultados podemos hablar de cambios similares a los que son habituales como consecuencia de procesos formativos, con magnitud cercana a ,30 en la competencia emocional total y en las dimensiones de regulación, autonomía y competencia social y cambios insignificantes o nulos por en cuanto a conciencia emocional o competencias de vida y bienestar.

\section{B. Impacto sobre la ansiedad}

De acuerdo con los resultados que podemos observar en la tabla 4, se ha producido una disminución en la ansiedad estado con un nivel de significación de $\mathrm{p}=, 003$ por lo que podemos afirmar que existen diferencias significativas entre el antes y el después de la participación en el campamento urbano en los niveles de ansiedad estado de los niños y niñas participantes. Estas diferencias son a favor de la situación final, es decir que ha habido una disminución de la ansiedad pasando de un nivel 27,31 a un nivel 25,71 .

Tabla 4. Cambios en la ansiedad entre la situación inicial y final

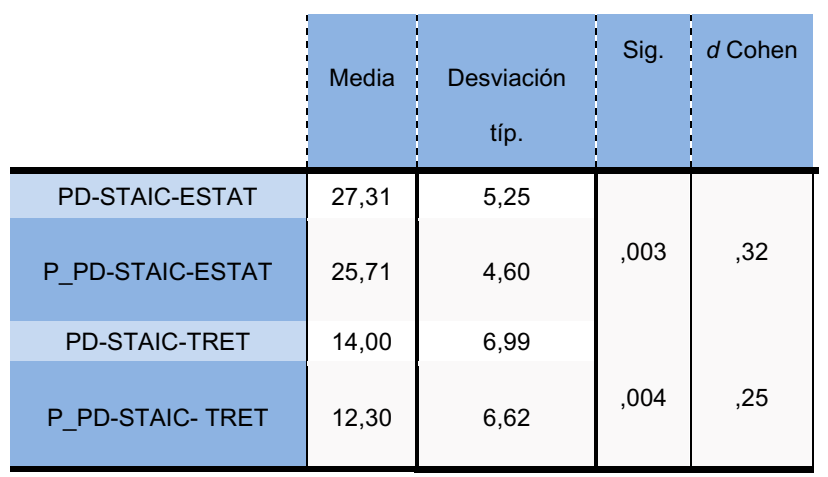

Cuando analizamos el progreso en la ansiedad rasgo vemos que curiosamente también se observa un cambio estadísticamente significativo con una probabilidad $\mathrm{p}=, 004 \mathrm{y}$ también a favor de la situación final, es decir indicando una disminución del valor inicial $(14,00)$ al final $(12,30)$, lo que significa una mejora en la ansiedad rasgo de los niños y niñas participantes.

Una vez detectadas las diferencias significativas se ha procedido a calcular la magnitud de estos cambios, para ello se ha estudiado la d de Cohen que se presenta en la Tabla 5. Observamos que podemos hablar de cambios muy similares a los que los que son habituales como consecuencia de procesos formativos, con magnitudes cercanas a 30.

\section{Percepción de los aprendizajes realizados y de su utilidad}

Las preguntas abiertas incluidas en el cuestionario se han procesado manualmente, ya que el volumen de datos era bastante manejable. Las respuestas han sido analizadas de forma iterativa, partiendo de la realidad o mundo interior de los menores, que se pone de manifiesto en sus respuestas. Es decir, hemos hecho un análisis de contenido manifiesto, inductivo. Este hecho es especialmente destacable, porque los grandes bloques y sus categorías han coincidido con el planteamiento de la intervención, el modelo teórico y los resultados del análisis cuantitativo.

La propuesta de categorización final se muestra en la tabla 5, además de las dos primeras columnas donde salen los bloques y sus categorías, en la tercera columna aparece la cantidad de niños y niñas que han dado un comentario relacionado con esta categoría. Se ha añadido una cuarta columna donde se ha hecho la correspondencia con la dimensión según el modelo teórico de desarrollo emocional.

Tabla 5. Categorización de preguntas abiertas

\begin{tabular}{|c|c|c|c|}
\hline Blocs & Categorías & $\begin{array}{c}\text { Frecuencia } \\
\text { de } \\
\text { aparición }\end{array}$ & $\begin{array}{c}\text { Dimensión } \\
\text { Modelo } \\
\text { teórico }\end{array}$ \\
\hline $\begin{array}{c}\text { Empoderamiento } \\
\text { personal }\end{array}$ & $\begin{array}{c}\text { gestión } \\
\text { emocional }\end{array}$ & 13 & Regulación \\
\hline
\end{tabular}




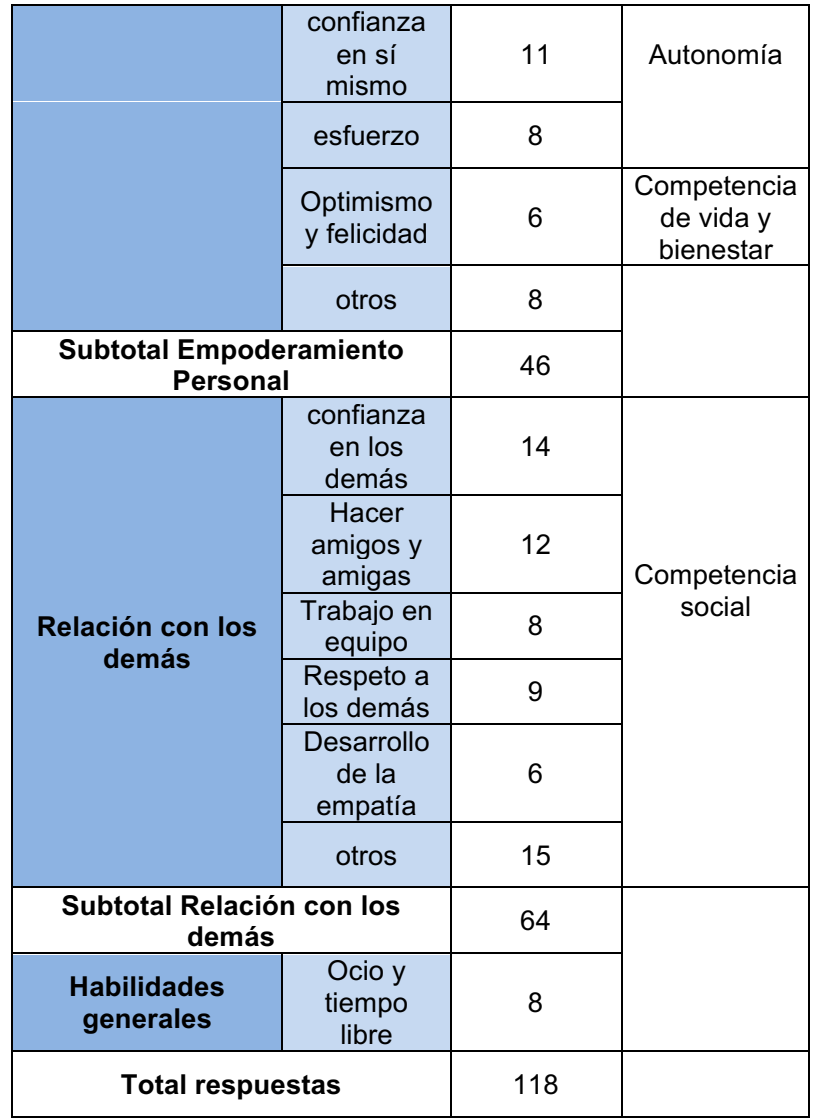

El bloque llamado Empoderamiento personal incluyen las respuestas que iban hacia el fortalecimiento personal, todas aquellas competencias emocionales que hacían referencia a la mejora de uno mismo, y que han acumulado 46 respuestas. Hacemos la transcripción de alguna respuesta especialmente significativa y que ilustra bien la categoría.

Ejemplo de respuesta relacionada con el proceso de regulación:

"He aprendido que cuando tengo miedo a alguna cosa la tengo que afrontar y no tener miedo" (Niña-9 años)

Ejemplo de comentario relacionado con la confianza en sí mismo y el esfuerzo:

“A ganar la confianza de mí mismo" (Niño-11años) Comentario relacionado con el optimismo y felicidad:

"He aprendido a vivir la vida con más optimismo, menos perfeccionismo y también a disfrutarla más" (Niño-9 años)

Bajo el bloque llamado relación con otras incluyen las respuestas que tenían que ver con el fortalecimiento de competencias emocionales que facilitan la creación de vínculos con los demás, en este caso se han acumulado 64 respuestas. Hacemos la transcripción de alguna respuesta especialmente significativa y que ilustra bien la categoría.
Ejemplo de respuesta relacionada con la confianza en los demás:

\section{"Si tú no confías en los demás, los demás no confiarán en ti.”(Niña-9 años)}

Ejemplo de comentario relacionado con hacer amigos y amigas: "Que hacer amigas es fácil cuando comienzas a
hablar con ellas" (Niña-9)

Ejemplo de respuestas relacionado con el trabajo en equipo:

"Lo que he aprendido en la granja y me será útil en mi vida es el trabajo en equipo." (Niño-12)

Comentario ejemplo del respeto a los demás:

"Yo este año he aprendido a tener más confianza conmigo misma, ser más flexible con la gente, y nunca faltar el respeto a mis compañeros y la gente de alrededor." (Niña-11)

Ejemplos de respuesta relacionadas con el desarrollo de la empatía:

"La verdad que he aprendido muchas cosas en La Granja porque llevo bastantes años aquí, pero puedo decir algunas cosas que he aprendido en el largo de estos años: se controlar más mi ira (que es muy fuerte), también he aprendido a enfrentarse a mis miedos y sacar mi valentía, también a ser más abierta y tener mucha más empatía de la que tengo." (Chica- 12 años)

"A confiar más, a empatizar con los compañeros, a
pensar qué puede pasar y ser más optimista." (Chico-12)

Bajo el bloque llamado Habilidades generales incluyen algunas respuestas (8) que no hacían referencia directa al desarrollo de competencias emocionales, y las hemos agrupado bajo este título ya que hablaban mayoritariamente del aprendizaje de la hípica, hacer cabañas con troncos dentro del bosque, y otros.

\section{"He aprendido a escalar, a ir en barca y construir} cabañas" (Chico-10)

\section{CONCLUSIONES}

En los últimos años se han multiplicado las acciones educativas para los períodos de vacaciones escolares y, entre ellas, los campamentos urbanos educativos son una de las fórmulas más populares. Hay muchos tipos de campamentos: deportivos (en una gran variedad de especialidades deportivas), multi-aventura, medioambientales, artísticos, históricoculturales y también algunos que pretenden el desarrollo de valores y competencias personales y relaciones interpersonales. 
En todos los casos se da por bueno que estos campamentos urbanos poseen efectos formativos en los niños, pero generalmente son actividades que a pesar de estar intencionalmente programadas no incluyen la evaluación de los aprendizajes producidos en los niños participantes, simplemente en algunas ocasiones se realiza una evaluación de la satisfacción de ellos o de sus familias.

En este estudio se ha puesto en evidencia que las actividades educativas en el tiempo libre pueden ser muy útiles para producir cambios en el desarrollo emocional de los niños y niñas.

El ambiente distendido, el compartir con otros chicos, el fijarse objetivos comunes y hacer esfuerzos conjuntos para alcanzarlos favorecen el desarrollo personal, la confianza en sí mismo y en los demás. La experiencia vivida permite una visión más global, y el desarrollo de la autonomía personal, además de facilitar el aprendizaje del amor y respeto por la naturaleza.

Estos resultados dan sentido al día a día en La Granja y coinciden con las valoraciones cualitativas relacionadas con la percepción de los aprendizajes y de su utilidad, de la mayoría de los 10.000 niños que, con sus escuelas, pasan por La Granja cada año disfrutando de las actividades Outdoor Training con caballos y animales de granja regladas y organizadas siguiendo el Método La Granja (Gutiérrez, 2014).

De acuerdo con la investigación efectuada, el uso del Método La Granja obtiene resultados significativos, tanto en el estudio cuantitativo como en el cualitativo, evidenciando el aprendizaje de competencias emocionales. Así, el Método La Granja puesto al servicio de la educación en el ámbito del ocio da como resultado el cambio significativo de varias competencias emocionales en los niños y jóvenes como por ejemplo que sean más conscientes de las emociones, que sienten sabiendo qué sirve cada emoción y poder regular (regulación emocional). La autonomía de los niños sale reforzada gracias al aumento de la seguridad y confianza en sí mismos que se trabaja intencionadamente durante las actividades, dándoles herramientas (paciencia para persistir y esforzarse) y recursos personales (regular la frustración) para hacer cosas que antes no hacían o que ni siquiera intentaban porque ellos creían que no podían, impactante positivamente en la autoestima. Respecto a las habilidades sociales: amigos, desarrollar la empatía, confiar en los demás, trabajar en equipo etc., son otros aspectos aprendidos y que les serán útiles en el futuro.

\section{REFERENCIAS}

Bisquerra, R. \& Pérez, N. (2007). Las competencias emocionales. Educación XXI, 10, 61-82.

Gutiérrez, Cristina (2014): Palabras de Niño. Santa Maria de Palautordera. Plataforma Editorial.

Krathwohl, D. (2002). A Revision of Bloom's Taxonomy: An Overview. Theory into Practice, 41(4), 212-218.

Mellado Santamaria, A. Y Mellado Santamaria, J.L. (2006) El campamento como medio educativo. Revista de Estudios de juventud, 72, 25-37.

Spielberger, C. D. (1989). Cuestionario de autoevaluación ansiedad estado-rasgo en ninos (STAI-C; $4^{\mathrm{a}}$ edición, 2009). Madrid, TEA Ediciones.

Trilla Bernet, Jaume (1997). La Educación Fuera de la Escuela. Madrid: Ariel. 